\title{
Fragments historiques d'une université à distance canadienne francophone : l'Université TÉLUQ Entretien avec Michel Umbriaco
}

\section{Historical Fragments of a French-speaking Canadian distance university: the Université TÉLUQ - Interview with Michel Umbriaco}

\section{Fragmentos históricos de una universidad canadiense de educación a distancia en francés: la Université TÉLUQ - Entrevista con Michel Umbriaco}

Cathia Papi, professeure

Université TÉLUQ, Canada

cathia.papi@teluq.ca

\section{RÉSUMÉ}

Dans cet entretien, Michel Umbriaco, qui a participé à la fondation de l'Université TÉLUQ, présente le contexte qui lui a donné naissance ainsi que quelques éléments ayant marqué son évolution. II explique que, créée au début des années 1970 en vue de favoriser l'accessibilité de l'éducation, cette université a traversé une succession de grands succès et de crises allant jusqu'à remettre en cause son existence. Après avoir ainsi parcouru le demi-siècle d'expérience de l'Université TÉLUQ, il évoque son avenir et les conditions de sa pérennité dans le contexte de l'enseignement supérieur au Québec et au Canada.

Mots-clés : formation à distance, enseignement supérieur, Québec, histoire, technologies éducatives

\section{ABSTRACT}

In this interview, Michel Umbriaco, who participated in the founding of Université TÉLUQ, presents the context that gave birth to the university and some of the elements that marked its evolution. He explains that created in the early 1970s to make education more accessible; this 
university has gone through a succession of great successes and crises that have even called its existence into question. After reviewing the half-century of experience of the Universite TÉLUQ, he discusses its future and the conditions for its continued existence in the context of higher education in Quebec and Canada.

Keywords: distance learning, higher education, Quebec, history, educational technologies

\section{RESUMEN}

En esta entrevista, Michel Umbriaco, que participó en la fundación de la Universidad TÉLUQ, presenta el contexto que dio origen a la universidad, así como algunos de los elementos que marcaron su evolución. Explica que, creada a principios de los años 1970 con el objetivo de hacer más accesible la educación, la universidad ha pasado por una sucesión de grandes éxitos y crisis que han llegado a cuestionar su existencia. Tras repasar el medio siglo de experiencia de TÉLUQ, analiza su futuro y las condiciones de su continuidad en el contexto de la enseñanza superior en Quebec y en Canadá.

Palabras clave: formación a distancia, educación superior, Québec, historia, tecnologías, educativas

Michel Umbriaco est professeur ${ }^{1}$ titulaire de cours de cycles supérieurs en gestion de l'éducation et en développement par la formation à distance au Département Éducation de l'Université TÉLUQ2', la seule université francophone entièrement à distance. II en est un des fondateurs et en est membre depuis près de cinquante ans. En plus d'avoir participé au développement de plus de quarante cours, il est l'auteur de nombreux documents et communications concernant la gestion et le financement des universités. II est le dernier professeur toujours en activité à pouvoir présenter l'évolution de l'Université TÉLUQ depuis sa création jusqu'à nos jours.

CATHIA PAPI : Michel, pourrais-tu nous retracer l'origine de I'Université TÉLUQ, initialement connue en tant que Télé-université de l'Université du Québec? Autrement dit, dans quel contexte a émergé l'idée de créer une université à distance? Quel était le public visé?

MICHEL UMBRIACO : Au tout début des années soixante-dix, quelques mois à peine après la création du réseau de l'Université du Québec, la réflexion s'est amorcée pour maximiser l'accessibilité de la population québécoise aux études universitaires. Un groupe mixte, gouvernement-université, a eu comme mission d'examiner les succès et les échecs d'expériences québécoises, canadiennes et internationales, telles que l'Open University du Royaume-Uni, l'University Without Walls américaines et des expériences comme TEVEC ${ }^{3}$ ou Multi-Média en éducation des adultes au Québec. Les promoteurs du projet de la Télé-université voulaient l'inscrire dans le vaste programme de démocratisation socioculturelle de

\footnotetext{
${ }^{1}$ Le générique masculin est utilisé dans ce document sans aucune discrimination et uniquement dans le but d'alléger le texte.

2 Pour simplifier la lecture, « Université TÉLUQ » est nommée « TÉLUQ » dans le reste du texte, sauf exception.

${ }^{3}$ TEVEC : Projet de télévision éducative (TEVEC) au Saguenay pour l'alphabétisation des adultes.
} 
l'université de la fin des années soixante. Donc, la toute première contribution de la TÉLUQ a été et demeure aujourd'hui l'accessibilité de la formation, ou du perfectionnement universitaire, à l'ensemble de la population québécoise ${ }^{4}$.

À l'époque, la formation à distance puisait trop souvent à la mode audiovisualiste et au behaviorisme en éducation hérité des recherches et pratiques de l'armée américaine. Ainsi, la technostructure (tous les services administratifs, de production matérielle, de soutien en technologie éducative, de fabrication et d'expédition du matériel des cours) représentait la grande majorité (plus de $85 \%$ ) des coûts et des efforts de développement, alors que les contenus de cours occupaient une portion congrue des ressources qui y étaient dédiées. Par ailleurs, déjà à cette période, il fallait répondre aux prophètes de mauvais augure qui affirmaient sans ambages que la formation à distance était une mode fort passagère qui mourrait à très court terme (" de six à neuf mois tout au plus » selon un recteur de l'époque). II fallait que l'équipe de fondation soit non seulement soudée, mais qu'elle ait une vision et une confiance en l'avenir qui dépassent les limites habituelles.

\section{CATHIA PAPI : Peux-tu nous dépeindre à quoi ressemblait la Télé-université à ses débuts? Qui en étaient les principaux acteurs? Quel était son mode de fonctionnement?}

MICHEL UMBRIACO : Comme dans plusieurs secteurs de la société occidentale, c'était l'époque des grandes espérances pour construire un monde meilleur. L'idée même de diplôme était contestée pour plutôt viser des apprentissages significatifs en vue du développement personnel de l'apprenant. Si tout le monde était épanoui et heureux, s'ensuivrait une société idéale.

Tout était organisé autour de projets assez indépendants les uns des autres et avec des équipes de projet largement conviviales et collaboratives. Ainsi, chaque cours produit devenait un événement majeur et public, car il s'agissait de faire en sorte que « les meilleurs esprits du Québec » puissent faire comprendre à la population ce qu'était sa propre condition, son environnement physique et socioéconomique, ses origines. C'était la période de ce que nous appelions les " grands cours-vedettes » comme Histoire du Québec, Économie, Coopération, Sexologie, Environnement, etc. Et des milliers de personnes se sont inscrites dans chacun de ces cours. En même temps, pour répondre à des besoins du gouvernement, la TÉLUQ a développé une offre de programmes de perfectionnement des maîtres: les programmes PERMAMA (Perfectionnement des maîtres en mathématiques) et PERMAFRA (Perfectionnement des maîtres en français) qui ont été des succès reconnus par le ministère de l'Éducation et la communauté enseignante.

Ces succès autant scientifiques, éducatifs que publics et financiers ont provoqué de l'envie de "petits esprits mesquins pseudo-universitaires $\|^{5}$ et, conséquemment, une série d'importantes crises institutionnelles.

\footnotetext{
${ }^{4}$ Une bonne partie de l'historique de la TÉLUQ a été relatée dans Umbriaco (2018).

${ }^{5}$ Expression du recteur d'une université canadienne.
} 
CATHIA PAPI : L'Université TÉLUQ est effectivement connue pour les différentes crises qui ont ponctué son histoire; pourrais-tu nous les rappeler brièvement et faire ressortir leur rôle dans l'évolution de l'établissement?

MICHEL UMBRIACO : Dans l'« histoire tumultueuse », comme le dit si bien Guillemet (2012), cité par le Conseil supérieur de l'éducation (2015, p. 44), de cette jeune organisation, il y a eu huit crises majeures qui l'ont menacée sérieusement de fermeture complète et définitive. On doit donc souligner que c'est le seul établissement universitaire du Québec et du Canada qui a fait les frais d'autant de " menaces ». Par ailleurs, cette succession de crises peut s'analyser en déclinant la succession des relations administratives et politiques des onze directions générales avec les gouvernements et les autres universités du Québec qui ont eu un impact majeur et déterminant sur la définition même de l'établissement. Se baser sur l'impact premier de ce groupe de onze leaders, c'est aussi souligner la relative fragilité de cette organisation, certes agile, mais aussi corollairement très vulnérable aux contextes sociopolitiques.

Force est de constater que ces crises, mis à part les changements de direction qu'elles ont souvent occasionnés, ont affiné la mission même de la TÉLUQ, ont encouragé son développement et lui ont permis de s'affirmer comme un établissement unique et essentiel dans le réseau universitaire canadien et international.

CATHIA PAPI : En dehors d'être intégralement à distance, quelles sont, selon toi, les spécificités de I'Université TÉLUQ relativement aux autres établissements d'enseignement supérieur québécois? Quelles sont également ses particularités relativement aux autres institutions internationales spécialisées en formation à distance telle que l'Athabasca University au Canada anglophone, l'Open University au Royaume-Uni ou le CNED en France?

MICHEL UMBRIACO: La grande différence, historique et organisationnelle, c'est que la TÉLUQ, contrairement à tous les autres établissements cités, n'a pas été planifiée ou créée par un gouvernement. C'est une création de l'Université du Québec. Si bien que ce n'est que vingt ans après sa création (en 1993) que le gouvernement reconnaissait le « potentiel et l'expertise unique de la TÉLUQ », lui octroyait une personnalité juridique en lui émettant des lettres patentes formelles.

Chacune des grandes institutions canadiennes et internationales a ses caractéristiques propres, mais toutes partagent de grandes similarités avec l'Université TÉLUQ, par exemple la spécialisation et la séparation des tâches d'enseignement entre plusieurs intervenants. En consultant les travaux et les publications d'éducation comparée, on peut aussi affirmer que la TÉLUQ tient davantage de la tradition anglo-saxonne de par son autonomie universitaire (liberté académique et autonomie institutionnelle).

CATHIA PAPI : Comment définirais-tu l'activité de professeur en formation à distance? Dans quelle mesure accompagne-t-il ses étudiants? Comment cette activité a-t-elle évoluée au cours du temps à l'Université TÉLUQ?

MICHEL UMBRIACO : C'est aux Comices ${ }^{6}$ de 1976, sous le leadership du directeur général de l'époque Fernand Grenier, que j'ai contribué à la "naissance » du corps professoral par la transformation de « quatre coordonnateurs pédagogiques de cours » en professeurs d'université, et ce, dans un climat

\footnotetext{
${ }^{6}$ Les Comices ont été la réunion, à l'été de 1976, de tous les artisans de la Télé-université pendant deux jours, à l'extérieur des locaux, pour constater collectivement ce que nous étions, et vers où nous désirions aller.
} 
d'opposition idéologique importante (plusieurs cadres et professionnels de la TÉLUQ ainsi que du réseau de l'Université du Québec arguaient ceci : « On n'a pas besoin de professeurs dans une Télé-université »), opposition qui a perduré de nombreuses années et dont on reconnaît encore des traces même aujourd'hui.

Comme on l'a souvent exprimé, il n'y a pas de différence radicale entre la formation à distance et la formation sur campus : ce sont les mêmes types d'acteurs (les professeurs, les étudiants et les autres intervenants pédagogiques). De plus, les relations fondamentales entre le Savoir, l'Inconnu et ceux qui s'y attèlent sont les mêmes. Ce ne sont donc que des modes différents d'apprentissage et de découverte entraînant des contraintes physiques, spatiales et temporelles différentes et donc, corollairement, des arrangements administratifs différents (politique, règles, procédures, etc.).

À ce dernier sujet, soulignons qu'une des avancées bénéfiques de l'éducation numérique de qualité prend forme dans une formalisation accrue et un investissement significatif (en temps et en spécialistes de soutien) à chacune des phases d'un cours ou d'un programme : planification et recherche pédagogique, conception (et design), production, implantation et diffusion, et enfin évaluation, rétroaction et recherche. On peut souligner aussi que la formation à distance et la technologie sont des enjeux du développement universitaire et, donc, que la formation à distance et la technologie sont des enjeux de négociations avec tous les corps d'emploi et, au premier chef, celle des professeurs qui doivent exercer un leadership académique.

CATHIA PAPI : La formation à distance offre, aux apprenants comme aux enseignants, une grande flexibilité. Elle fait appel à leur autonomie et à leur capacité de régulation de leurs activités. À la lumière de ton expérience, aurais-tu des conseils à donner aux professeurs, voire aux étudiants, qui actuellement découvrent la formation à distance dans ce contexte de pandémie?

MICHEL UMBRIACO : Faites-vous confiance et soyez patients avec vos collègues et vous-même. S'initier à la formation à distance (ou à toute autre transformation) en période de crise est rarement une bonne idée. Trop de prophètes, ou trop de personnes négatives, trop d'apprentis sorciers font qu'on semble oublier, sous prétexte de l'urgence, une très grande partie des bonnes pratiques en formation à distance et en apprentissage numérique.

Pour comprendre mon propos, il suffit d'un seul exemple : dès les débuts de l'enseignement à distance au Québec, dans les années soixante-dix, la nécessité d'une convivialité entre les personnes et d'un contact personnalisé a été mis en évidence par plusieurs recherches (entre autres celles de l'INRS, du GIREFAD, du LICEF ${ }^{7}$, etc.) comme condition nécessaire à la réussite et à la persévérance aux études. D'ailleurs, les résultats récents révèlent bien que la formule d'apprentissage autonome se voit grandement bonifiée lorsqu'on y insère des activités d'intégration interactives en groupe.

Par ailleurs, au cours des années, nous avons élaboré, au GIREFAD et dans les équipes de développement de programmes, un certain nombre de maximes inspirant encore nos actions :

- Toute action vaut mieux que n'importe quelle critique;

- La bonne volonté ne peut pas remplacer la compétence;

- Ainsi le règne de l'approximation est à proscrire;

- Il faut travailler avec les communautés afin d'éviter le « dumping » éducatif ou culturel;

\footnotetext{
${ }^{7}$ INRS : Institut national de la recherche scientifique;

GIREFAD : Groupe interinstitutionnel de recherche en formation à distance;

LICEF : Laboratoire d'informatique cognitive et environnements de formation.
} 
- On peut toujours faire des expériences et «tester » des solutions originales, mais il ne faut surtout pas les « essayer » à grande échelle ni les exporter;

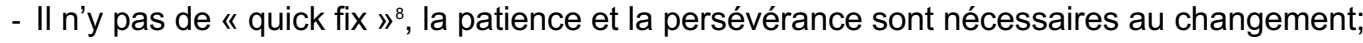

- II faut rester optimiste face au développement.

CATHIA PAPI : De façon plus générale, en tant qu'être humain, que t'a apporté ta carrière à I'Université TÉLUQ? Quels sont tes plus grands accomplissements? Quels sont les événements qui t'ont marqué positivement ou négativement?

MICHEL UMBRIACO : J'ai commencé à m'intéresser à la formation à distance et aux travaux qui ont mené à la création de la TÉLUQ au tout début des années soixante-dix. Aujourd'hui, déjà cinquante ans plus tard, la TÉLUQ a été et est encore l'axe central de ma vie professionnelle. Résumer ce parcours est un défi marqué par l'impossibilité matérielle de traquer, pour soi et pour les autres, l'incommensurable d'une vie professionnelle. En effet, une " carrière » ne peut s'inscrire que dans un maelström de choix individuels dépendant des contextes scientifiques, sociaux, psychiques et politiques particuliers, et évidemment changeants. Malgré tout, l'élément cardinal de toute cette carrière s'inscrit dans un désir et une volonté de développer la formation à distance pour répondre aux besoins d'accessibilité universitaire de la population québécoise. Pour ce faire, au cours des années, j'ai travaillé à plus de quarante cours et à une trentaine de projets de formation continue; la partie la plus réjouissante de ces nombreux chantiers, ce sont les témoignages des étudiants ou des clients pour qui la TÉLUQ a fait une différence dans leur vie.

CATHIA PAPI : Depuis plusieurs années, tu travailles en prospective de l'enseignement supérieur avec la FQPPU ${ }^{9}$ et, plus récemment, avec le groupe présidé par Rémi Quirion, le scientifique en chef du Québec, sur l'université québécoise du futur. À partir de ces travaux, quel avenir entrevoistu pour la TÉLUQ?

MICHEL UMBRIACO : En effet, le groupe de travail, présidé par le scientifique en chef, a remis, le $1^{\text {er }}$ décembre dernier, un rapport d'une centaine de pages, intitulé L'université québécoise du futur: Tendances, enjeux, pistes d'action et recommandations, à la ministre de l'Enseignement supérieur (Quirion, 2020). La ministre devait le rendre public au début de février. On se doit de constater que la très grande majorité des principaux enjeux nationaux et internationaux contenus dans ce rapport, tels que la crise climatique, les pandémies, les grandes menaces économiques et démographiques, le déploiement du numérique, la mondialisation, etc. sont au cœur des projets de recherche et de l'enseignement de plusieurs équipes de professeurs de la TÉLUQ.

Quant à la TÉLUQ, elle doit, selon moi, rester au centre, en collaboration avec les collègues des autres universités, de toute stratégie numérique des gouvernements du Canada et du Québec, incluant les initiatives collectives concernant le e-learning. Par ailleurs, comme cela a été évoqué aux consultations gouvernementales sur l'avenir de l'université de l'automne dernier, le « numérique modifie tout et partout », si bien qu'on réclame des universités et de la TÉLUQ qu'elles proposent des outils pour développer « un solide sens critique pour éviter l'enivrement par la technologie ». En effet, « le numérique ne doit pas nous imposer des changements, mais bien demeurer un outil de changement » (Quirion, 2020, p. 14).

\footnotetext{
${ }^{8}$ Solution miracle.

${ }^{9}$ Fédération québécoise des professeures et professeurs d'université (FQPPU).
} 
CATHIA PAPI : Pour conclure, avec le développement conséquent de la formation à distance depuis l'essor d'Internet et encore plus fortement depuis le début de la pandémie, l'Université TÉLUQ a-t-elle encore sa raison d'être? Autrement dit, si tu en connais bien le passé, quel avenir vois-tu se profiler à l'horizon pour cette université bien particulière?

MICHEL UMBRIACO : Tous ceux qui s'intéressent au changement savent bien que le passé n'est jamais garant de l'avenir. Par ailleurs, regarder l'histoire reste un exercice toujours un peu difficile parce que même si on peut rationaliser ex post pour y trouver une cohérence, on sait, surtout sur une période de près de cinquante ans, qu'elle est plutôt marquée de mouvements stochastiques.

On a souvent prédit la fin de la TÉLUQ, en prétextant de sa non-pertinence, ou de sa lourdeur, ou encore de sa petitesse, ou de son manque de crédibilité académique et que sais-je encore, et ce, dès 1973. Mais force est de constater qu'elle est toujours là à rendre des services qu'elle seule encore peut rendre ${ }^{10}$. Son avenir, comme pour la majorité des institutions, dépendra essentiellement de la passion de ses artisans à promouvoir de manière cohérente et efficace des valeurs d'accessibilité et d'ouverture.

\section{Liste de références}

Conseil supérieur de l'éducation. (2015). La formation à distance dans les universités québécoises : un potentiel à optimiser. Avis au ministre de l'Éducation, de l'Enseignement supérieur et de la Recherche. Gouvernement du Québec. https://www.cse.gouv.qc.ca/publications/formation-distance-universites-50-0486/

Guillemet, P. (2012). D'une université nouvelle à l'autre : la TÉLUQ et l'université bimodale. Dans L. Roy et Y. Gingras (dir.), Les universités nouvelles : enjeux et perspectives. Presses de l'Université du Québec, p. 259-276.

Quirion R. (2020, septembre). L'université québécoise du futur : Tendances, enjeux, pistes d'action et recommandations. Document de réflexion et de consultation, Fonds de recherche du Québec. http://www.scientifique-enchef.gouv.qc.ca/wp-content/uploads/UduFutur-FRQ-1.pdf

Umbriaco, M. (2018). Chapitre 6. Une analyse historique de la formation à distance à la Télé-université, Dans $P$. Doray, E.L. Dussault, Y. Rousseau et L. Sauvageau, L'Université du Québec 1968-2018 : 50 ans de contributions éducatives et scientifiques au développement du Québec (p. 115-126). Presses de l'Université du Québec.

\footnotetext{
${ }^{10}$ À titre d'exemple, la TÉLUQ a développé, à l'été 2020, à la demande du gouvernement du Québec, la formation à distance « J'enseigne à distance ", destinée aux enseignants de l'ensemble des ordres d'enseignement. https://www.teluq.ca/site/etudes/clom/enseigne-adistance.php
} 